\title{
A MATLAB-based PMU Simulator
}

\author{
Daniel Dotta, Member, IEEE, Joe H. Chow, Fellow, IEEE, Luigi Vanfretti, Member, IEEE \\ Muhammad S. Almas, Student Member, IEEE, and Marcelo N. Agostini, Member, IEEE
}

\begin{abstract}
The use of Phasor Measurement Unit (PMU) data in power system operation is of practical importance. These data are currently used for real-time operation monitoring and offline analysis. Understanding of the mechanisms involved in the phasor estimation process is necessary for correct phasor data interpretation and analysis, as well as for design of advanced control and protection schemes. In this paper the main phenomena involved in the phasor measurement process are illustrated using a MATLAB based PMU simulator.
\end{abstract}

Index Terms-PMU, phasor data processing, power system analysis, phasor estimation algorithms.

\section{INTRODUCTION}

$\mathbf{N}$ Owadays Wide Area Measurement Systems (WAMS) are being built around the world [1, 2, 3]. Despite the different development stages and applications found in different countries, the Independent System Operators (ISOs) recognize this technology's potential. WAMS are composed of Phasor Measurement Units (PMUs), high-speed communication channels and Phasor Data Concentrators (PDCs) [4]. The main idea is to measure three phase voltage and current waveforms, to estimated phasors synchronized with the Global Positioning System (GPS) using these waveforms and to send the resulting data to a central location (substation or Control Center) where the PDC is located.

In recent years, significant research effort was dedicated to develop applications using phasor measurement data. In particular, PMUs became an indispensable tool for postmortem analysis [5]. Some examples can be found in Brazilian [6] and Colombian blackouts [7].

Despite the great level of the research done in the PMU applications, a detailed understanding of the phasor estimation process could be helpful. Compared to the traditional measurement devices, like analog current and voltage oscillography, or digital devices, like digital disturbance recorders (DDR) (where the voltage/current waveforms are directly measured and converted to digital data), the PMU device uses signal processing techniques not familiar to every power system engineer. The approach to carry out this process in estimating phasors may raise concerns for the use of PMU data for advanced control and protection applications, as well as the interpretation of the data measured for real-time monitoring and off-line analysis. Additionally, WAMS provide the base platform for advance control and protection applications necessary for the future smart transmission grids. To reach this

Daniel Dotta is with the Federal Institute of Santa Catarina, Florianópolis, SC, Brazil. (email:dotta@ifsc.edu.br). J.H. Chow is with Rensselaer Polytechnic Institute, Troy, NY, US. (email:chowj@rpi.edu). L. Vanfretti and M. S. Almas are with KTH Royal Institute of Technology, Stockholm, Sweden. (email:\{luigiv,msalmas\}@kth.se) goal the PMU network and the measurement process need to be clear and easily understandable, in order for the power system engineers to feel confident about using this data for advanced applications.

The aim of this article is to present a MATLAB software that can be used as a teaching tool and research framework to explore the algorithms involved in the phasor measurement process. This software can process simulated and real measured signals. The main idea is to provide a software tool to better understand the phenomena involved in the phasor measurement process, such as off-nominal frequency and unbalanced frequency operation, the influence of the complex gains $P_{n}$ and $Q_{n}$ in the process, and the methods used to minimize their influence.

The paper is organized as follows. In Section II, PMU architectures and the phasor measurement process are presented. In Section III a MATLAB based simulator is presented. In Section IV results of the proposed approach are presented. The conclusions are presented in Section V.

\section{Phasor Measurements Architectures}

Figure 1 illustrates the two basic commercial PMU architectures available [8]. These schemes are necessary to reduce the effect caused by leakage phenomena. The basic block diagrams are the same and can be divided in:

- Sampling and filtering;

- Frequency and Phasor (Discrete Fourier Transform DFT) estimators.

The main difference between the architectures is in the way the signal is sampled:

- Uniform (fixed) sampling rate;

- Non-uniform (variable) sampling rate.

The first architecture was the first used because uniform sampling simplifies the acquisition process and the signal processing tasks. Previous works are dedicated to exploring and improving uniform sampling methodologies, presented in [4], [5], [9], [10], [11] and [12]. Since uniform sampling is well described in the literature, it will be the main architecture to be explored in this paper.

Non-uniform methodologies are not well explored in the literature. From the first published work [13], a few papers have been published regarding it [11]; and some US patents are found in [14] [15]. The main technical issue is to relate the time-tag given by the GPS clock to the sampling clock generated by the local power system frequency measurement.

\section{A. Uniform Sampling Phasor Measurement Processing}

The uniform sampling phasor measurement process is divided in three main parts: phasor estimation using (recursive 

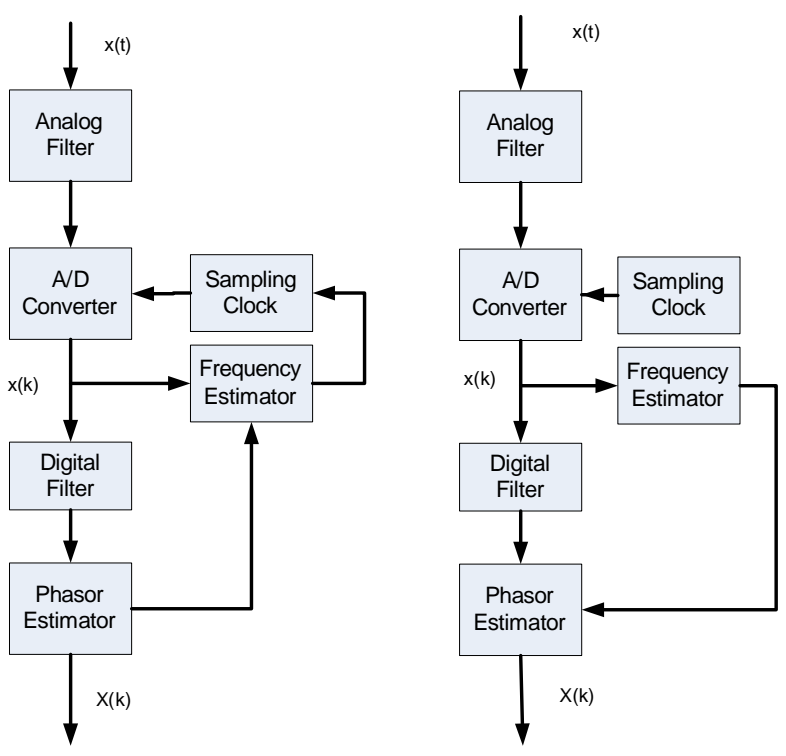

Fig. 1. Basic Phasor estimation architectures [8]

or nonrecursive) discrete Fourier Transform (DFT), frequency estimation, and post-processing (using calibration factors and filtering), as shown in Figure 2. Under off-nominal frequency operation, the post-processing layer is necessary to correct the effects caused by leakage phenomena. Leakage phenomena results from the truncation of sampled data outside the data window. Consequently, the estimated phasor is attenuated by two complex gains, $P_{n}$ and $Q_{n}{ }^{1}$. Fortunately, the effects of the complex gain $P_{n}$ (shown in Figure 2) can be readily computed from the sampling window size $(N)$, the frequency deviation $(\Delta \omega)$ and the sampling period $(\Delta t)$ [4]. The magnitude of $P_{n}$ is the attenuation factor, and the phase angle of $P_{n}$ is a constant offset in the measured phase angles. As the window size $(N)$ and sampling period $(\triangle t)$ are fixed, $P_{n}$ can be readily estimated for a frequency range and stored in a table (Block 1 in Figure 2). In real-time, the frequency deviation estimation is necessary to take the correct $P_{n}$ value.

The complex gain

$$
Q=\left\{\frac{\sin \frac{N\left(\omega+\omega_{0}\right) \Delta t}{2}}{N \sin \frac{\left(\omega+\omega_{0}\right) \Delta t}{2}}\right\} e^{-j(N-1) \frac{\left(\omega+\omega_{0}\right) \Delta t}{2}}
$$

introduces a magnitude and phase angle variation at frequency $2 \omega_{0}+\Delta \omega \simeq 2 \omega_{0}$ (approximately) in the estimated singlephase phasor. The second harmonic $\left(2 \omega_{0}\right)$ oscillation is shown in Figure 7 (blue curve). In contrast to a static offset, this oscillation is not easily removed. A conventional way to minimize its influence is to use a three-point-average filter (Block 2 in Figure 2) [4], which can reduce the harmonic components by more than $50 \%$.

1) Frequency Estimation: During normal operating conditions, the power system the frequency is always changing. This deviation can be small, when related to generation load mismatch, or large, when considering large disturbances. Under these conditions, the frequency estimation methodology bears a key role in the phasor computation process. An interesting

$$
{ }^{1} X^{e s t}=P X^{\text {true }}+Q\left(X^{\text {true }}\right)^{*}[4] .
$$

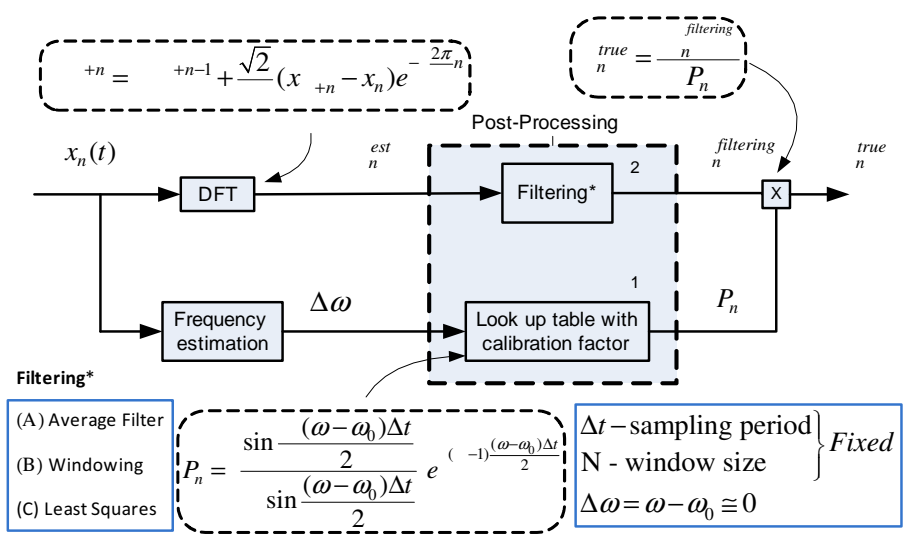

Fig. 2. Phasor processing algorithm for uniform sampling [5]

overview of the power system frequency concept is found in [8].

Several frequency measurement methodologies can be found in the literature. An evaluation of the frequency estimation methodologies can be found in $[16,17,18,19,20,21$, 22, 23]. The main available methods are: Zero Crossing [17], Least Error Squares [24], Kalman filters [25], Demodulation [18], [22], Phasor-Based [17],[20],[21].

The methods used in the PMU Simulator are Demodulation and Phasor-Based. These methods are chosen because they presented satisfactory performance under large frequency variation and noisy environment, and are built into commercial PMUs (phasor-based).

\section{Matlab-based PMU Simulator}

The software is suitable to explore the phasor measurements estimation process described in Figure 2. The MATLAB PMU simulator considers single-phase and three-phase measurement signals, the last one allowing positive sequence phasor estimation. Step and ramp (frequency modulation) frequency disturbances can be introduced. Simulations can be realized considering off-nominal frequency operation to observe the influence of the complex gains $\left(P_{n}\right.$ and $\left.Q_{n}\right)$ and filtering in the phasor estimation process. The processing of real digital measurement data can also be realized. The main software blocks and algorithms involved in the MATLAB PMU simulator are shown in Figure 3.

The main features of the PMU Simulator are:

- Recursive and Non-Recursive DFT;

- Off-nominal frequency simulation, frequency step and ramp;

- Influence of the complex gains in the phasor measurement;

- Influence of the post-processing level in the phasor measurement;

- Real digital data processing.

\section{Simulation Results}

The main goal of this section is to describe the performance of three-phase and single-phase phasor estimation under offnominal frequency operation, using simulated data. To illustrate the phasor performance a frequency step disturbance 


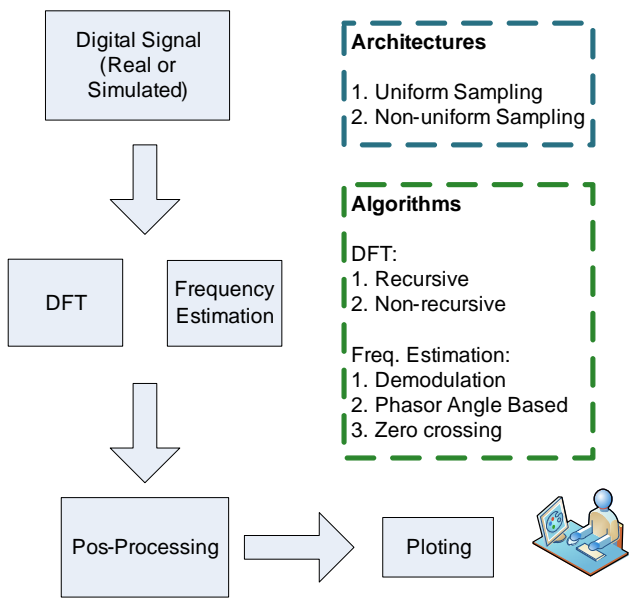

Fig. 3. Software Diagram
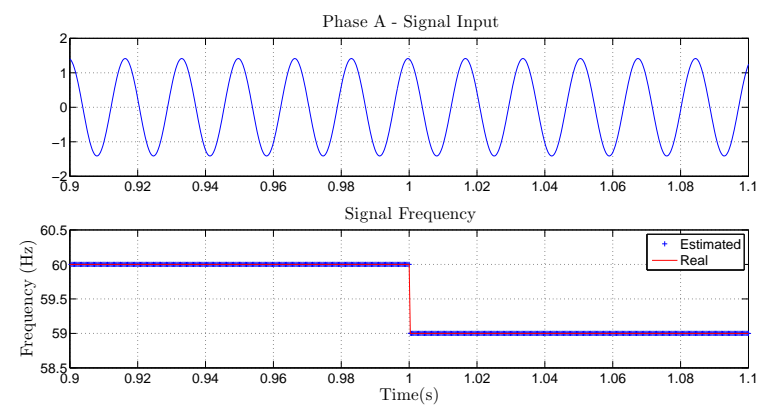

Fig. 4. Input Signal - Sine Wave

is applied, after $1 s$, in the sine wave signal source. This disturbance is show in Figure 4. The non-recursive DFT and demodulation algorithms are, respectively, the estimated phasor and frequency. The window size is set to 48 points per cycle, that is, a sampling rate of $2.88 \mathrm{kHz}$.

\section{A. Three-Phase Performance}

The influence of the complex gains $P_{n}$ and $Q_{n}$ in the three-phase phasor measurements under off-nominal frequency operation is shown in Figure 5.

The simulation in Figure 5 shows the influence of the complex gain $P_{n}$ in the phasor magnitude and phase. It should be noted that the influence of the complex gain $Q_{n}$, a second harmonic oscillation, is filtered by the three-phase estimation.

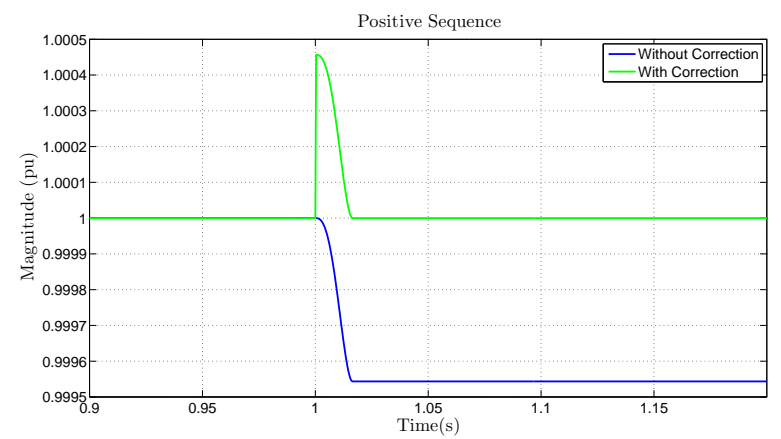

Fig. 5. Three-Phase Positive Sequence Performance

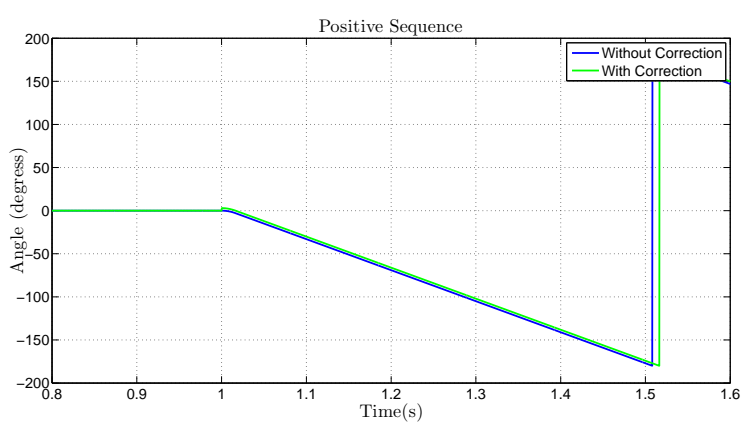

Fig. 6. Three-Phase Positive Sequence Performance

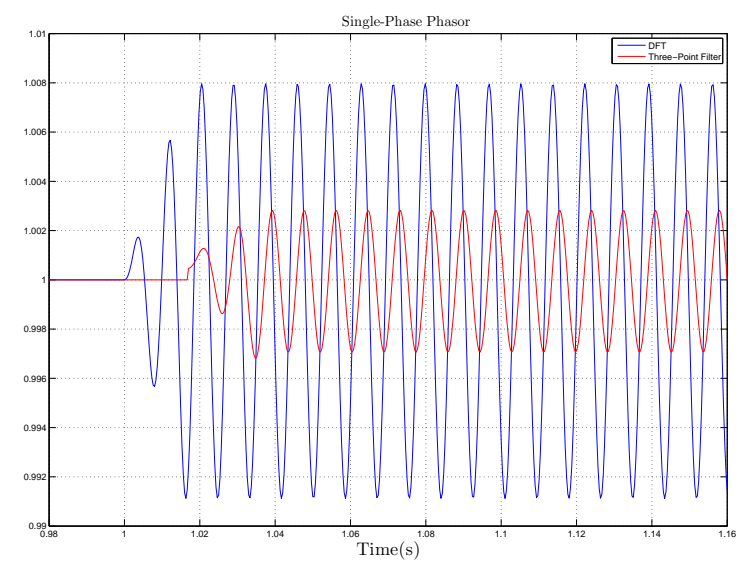

Fig. 7. Single-Phase Phasor Estimation Performance

\section{B. Single-Phase Performance}

The performance of the single-phase phasor estimation under off-nominal frequency is shown in Figure 7.

In this simulation, the influence of the complex gains $P_{n}$ andn $Q_{n}$ under off-nominal frequency operation is clearly revealed. The average three-point filter reduces the influence of the second harmonic however a small delay is found in the measurement. The phasor angle is shown in Figure 8.

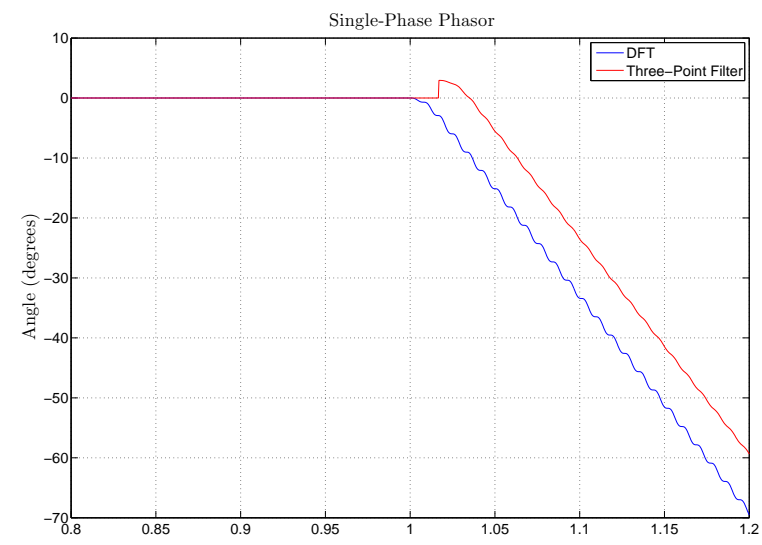

Fig. 8. Single-Phase Phasor Estimation Performance 


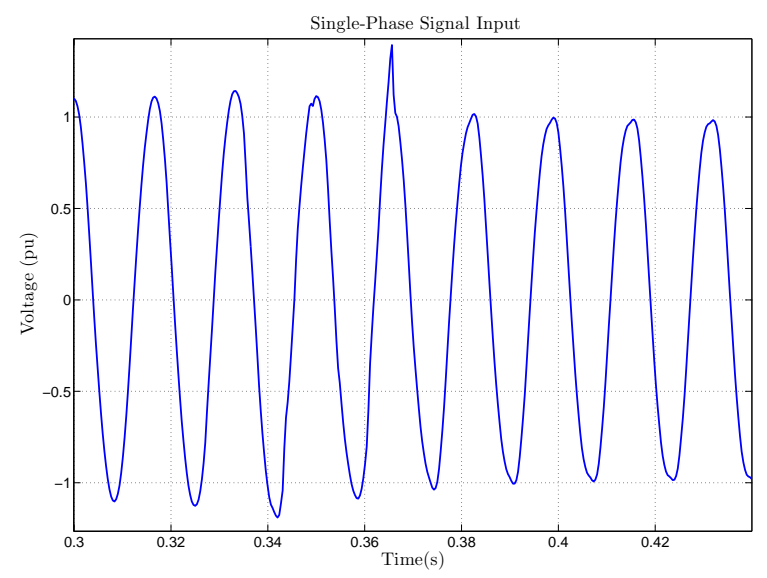

Fig. 9. Single-Phase Real Data

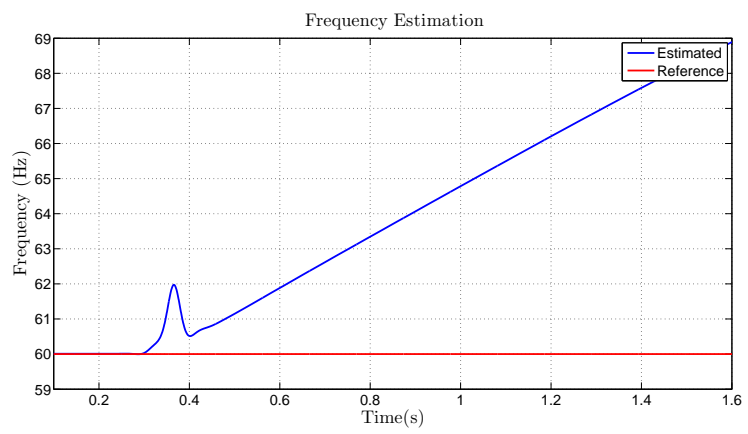

Fig. 10. Single-Phase Real Data

\section{Real Data}

Real digital data files can be also processed by the PMU Simulator. In this case, three-phase real digital data acquired during a Hydro Generator Load Rejection Test is processed by the simulator. The hydro unit data was recorded at 48 points per cycle, that is, a sampling rate of $2.88 \mathrm{kHz}$. The voltage data acquired and the frequency estimation during the fault are shown in Figures 9 and 10, respectively.

It should be noted that the load rejection happened around 0.35 s., the voltage wave presents some spikes related with the circuit breaker operation. The frequency estimation before and after the disturbance is shown in Figure 10.

Figure 10 shows the frequency behavior under disturbance. Following the load rejection, the frequency rises significantly because the generator is operating with no load. As expected there is a delay related to the actuation of the speed governor.

The positive sequence voltage magnitude from the phasor estimation is presented in Figure 11.

Figure 11 clearly shows the influence of the complex gain $P_{n}$ as well as a small oscillation, probably related with a small unbalances between the generator phases. The phasor angle is shown in the Figure 12.

\section{CONClusion}

In this paper a PMU MATLAB simulator was presented. The aim of this software is to aid in the understanding of the behaviour of algorithms internal to the PMU and

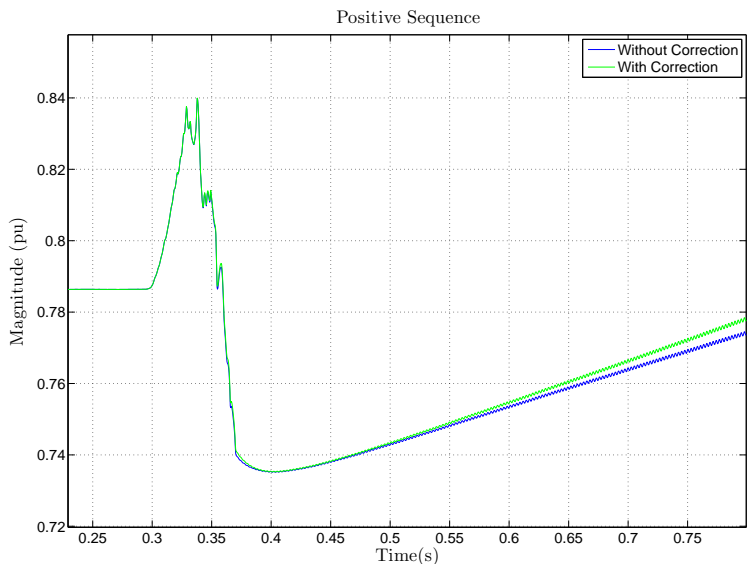

Fig. 11. Single-Phase Real Data

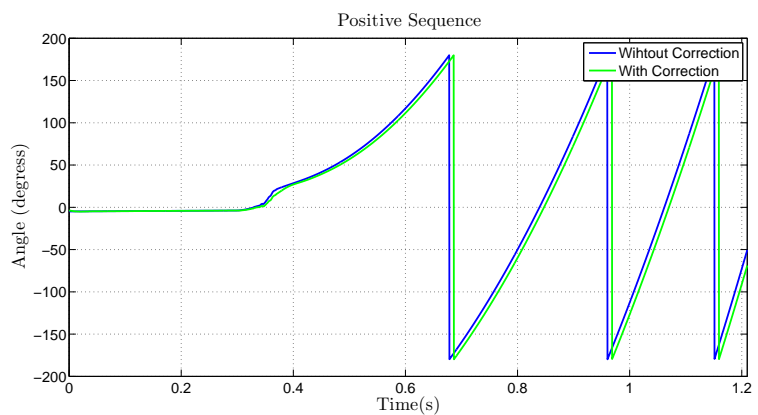

Fig. 12. Single-Phase Real Data

to grasp key factors affecting their performance under offnominal frequency operation. The PMU simulator is useful for academic and professionals who would like to understand the concepts involved in the phasor estimation process carried out by PMUs. The performance of the simulator was evaluated using simulated and real data from $2.88 \mathrm{kHz}$ measurements. The output of the estimation process was compared with reference traces and phasors from real PMUs. The next step in the development of the MATLAB-based PMU simulator is to implement the non-uniform sampling architecture.

\section{ACKNOWLEDGMENT}

The authors gratefully acknowledge the financial support of the Brazilian Government Research Agency (CNPq) process number 201249/2011-1 and Federal Institute of Santa Catarina (IFSC).

L. Vanfretti is supported by the European Commission within the FP7 $i$ Tesla project and the STandUP for Energy collaboration initiative. M.S. Almas is supported by NER through the STRON $g^{2}$ rid project.

\section{REFERENCES}

[1] I. Kamwa, R. Grondin, and Y. Hebert, "Wide-area measurement based stabilizing control of large power systems-a decentralized/hierarchical approach," IEEE Trans. Power Syst., vol. 16, no. 1, pp. 136-153, 2001.

[2] I. C. Decker, D. Dotta, M. N. Agostini, S. L. Zimath, and A. S. de Silva, "Performance of a synchronized phasor 
measurements system in the brazilian power system," in IEEE Power Eng. Society General Meeting, 2006.

[3] X. Xie, Y. Xin, J. Xiao, J. Wu, and Y. Han, "WAMS applications in Chinese power systems," IEEE Power Energy Mag., vol. 4, no. 1, pp. 54-63, 2006.

[4] A. G. Phadke and J. S. Thorp, Synchronized phasor measurements and their applications. New York: Springer, 2008.

[5] M. Akke and J. S. Thorp, "Sample value adjustment improves phasor estimation at off-nominal frequencies," IEEE Trans. on Power Delivery, vol. 25, no. 4, pp. 22552263, 2010.

[6] I. C. Decker et al., "Monitoring of a large scale event in the Brazilian power system by WAMS," in Proc. VIII iREP, 2010, pp. 1-8.

[7] C. Ruiz, N. Orrego, and J. Gutierrez, "The Colombian 2007 black out," in Trans. and Distr. Conf. and Exposition: Latin America, 2008 IEEE/PES, 2008.

[8] A. G. Phadke and B. Kasztenny, "Synchronized phasor and frequency measurement under transient conditions," IEEE Trans. Power Del., vol. 24, no. 1, pp. 89-95, 2009.

[9] M. Paolone, A. Borghetti, and C. A. Nucci, "A synchrophasor estimation algorithm for the monitoring of active distribution newtorks in steady state and transient conditions," in Proc. 17th Power systems Computation Conference, 2011, pp. 1-8.

[10] T. Nguyen and X. Li, "A fast and accurate method for estimating power systems phasors using dft with interpolation," in PES General Meeting, 2006. IEEE, 0-0 2006, p. 8 pp.

[11] W. Premerlani, B. Kasztenny, and M. Adamiak, "Development and implementation of a synchrophasor estimator capable of measurements under dynamic conditions," Power Delivery, IEEE Transactions on, vol. 23, no. 1, pp. $109-123$, jan. 2008.

[12] J. de la O Serna, "Dynamic phasor estimates for power system oscillations and transient detection," in Power Engineering Society General Meeting, 2006. IEEE, 0-0 2006, p. 7 pp.

[13] G. Benmouyal, "Design of a combined digital global differential and volt/hertz relay for step-up transformers," IEEE Trans. Power Del., vol. 6, no. 3, pp. 1000-1007, 1991.

[14] L. S. A. G. C. Zweigle and A. Guzman-Casillas, "Tapparatus and algorithm for estimating synchronized phasors at pre-determined times referenced to an absolute time standard in an electrical system," US Patent 7480 580, 20, 2009.

[15] G. C. Z. L. R. S. Anderson, A. Guzman-Casillas and G. Ben, "Protective relay with synchronized phasor at pre-determined time referencedto an absolute time standard in an electrical sys," US Patent 6845333, 2005.

[16] D. W. P. Thomas and M. S. Woolfson, "Evaluation of frequency tracking methods," IEEE Trans. on Power Delivery, vol. 16, no. 3, pp. 367-371, 2001.

[17] M. M. Begovic, P. M. Djuric, S. Dunlap, and A. G. Phadke, "Frequency tracking in power networks in the presence of harmonics," IEEE Trans. Power Del., vol. 8, no. 2, pp. 480-486, 1993.

[18] M. Akke, "Frequency estimation by demodulation of two complex signals," IEEE Trans. Power Del., vol. 12, no. 1, pp. 157-163, 1997.

[19] I. Kamwa, M. Leclerc, and D. McNabb, "Performance of demodulation-based frequency measurement algorithms used in typical pmus," IEEE Trans. Power Del., vol. 19, no. 2, pp. 505-514, 2004.

[20] D. Fan and V. Centeno, "Phasor-based synchronized frequency measurement in power systems," IEEE Trans. Power Del., vol. 22, no. 4, pp. 2010-2016, 2007.

[21] — , "Least-squares estimation in phasor-based synchronized frequency measurements," in Proc. IEEE Power and Energy Society General Meeting - Conversion and Delivery of Electrical Energy in the 21st Century, 2008, pp. 1-6.

[22] T. S. J. Warichet and J. C. Maun, "Considerations about synchrophasors measurement in dymanic system conditions," Electrical Power and Energy Systems, vol. 31, no. 9, pp. 452-464, October 2009.

[23] S. N. et al., "Fundamental frequency estimation in power systems using complex prony analysis," Journal of Electrical Engineering \& Technology, vol. 6, no. 2, pp. 154 160, 2011.

[24] M. M. Giray and M. S. Sachdev, "Off-nominal frequency measurements in electric power systems," IEEE Trans. Power Del., vol. 4, no. 3, pp. 1573-1578, 1989.

[25] A. A. Girgis and T. L. D. Hwang, "Optimal estimation of voltage phasors and frequency deviation using linear and nonlinear kalman filtering: Theory and limitations," IEEE Power Eng. Rev., no. 10, pp. 46-47, 1984.

Daniel Dotta received his MSc. Degree in Electrical Engineering in 2003, and the PhD Degree in Power System Engineering in 2009, both from the Federal University of Santa Catarina, Florianpolis, Brazil. He has been on the faculty at the Federal Institute of Santa Catarina since 2006. Currently he is doing his sabbatical at Renssenlaer Polytechnic Institute (RPI), Troy, NY.

Joe H. Chow received his $\mathrm{MS}$ and $\mathrm{PhD}$ degrees from the University of Illinois, Urbana-Champaign. After working in the General Electric power system business in Schenectady, he joined Rensselaer Polytechnic Institute in 1987, and is a professor of Electrical, Computer, and Systems Engineering. His research interests include multivariable control, power system dynamics and control, voltage-source converter-based FACTS controllers, and synchronized phasor data.

Luigi Vanfretti (Student Member '03, M'10) became an Assistant Professor at the Electric Power Systems Department, KTH Royal Institute of Technology, Stockholm, Sweden, in 2010 and was conferred the Swedish academic title of Docent in 2012. He received his MSc in 2007 and $\mathrm{PhD}$ in 2009, both in Electric Power Engineering, from Rensselaer Polytechnic Institute (RPI), Troy, NY, USA. His research interests are in the general area of modeling, dynamics, stability and control of power systems; while his main focus is on the development of applications of PMU data.

M. Shoaib Almas obtained the MSc in Electric Power Engineering from KTH Royal Institute of Technology, Stockholm Sweden in 2011 where he is now a PhD Student. He obtained the BSc in Electrical Engineering from NUST, Pakistan. He has professional experience in substation automation and protection. 